\title{
MAGNETIC AND MAGNETO-OPTICAL PROPERTIES OF PrGd/FeCo MULTILAYERS
}

\author{
H. WATABE, Y. FUJIWARA, X. Y. YU, S. IWATA, S. TSUNASHIMA and S. UCHIYAMA* \\ Department of Electronics, Nagoya University, Nagoya 464-01, Japan \\ *Dept. of Information Network Eng., Aichi Inst. Technol., Toyota 470-03, Japan
}

\begin{abstract}
PrGd/FeCo multilayers prepared by the rf sputtering method exhibit perpendicular magnetic anisotropy $\mathrm{Ku}$ enhanced by the multilayer (ML) structure. The largest value of $\mathrm{Ku}$ obtained is about 30 $\times 10^{4} \mathrm{~J} / \mathrm{m}^{3}$ at a bilayer period of $1 \mathrm{~nm}$ with Pr composition at around 35at.\% in rare earth layers and Co composition at 10 at.\% in transition metal layers. The figure of merits of magneto-optical effect, is 20 to $30 \%$ larger than conventional TbFeCo films at the wavelength of $400 \mathrm{~nm}$. From the analysis of low angle $\mathrm{X}$-ray diffraction, the relationship between the enhanced anisotropy and the ML structure is discussed. KEYWORDS: MAGNETO-OPTICAL DISKS, RARE EARTH-TRANSITION, MULTILAYERS, MAGNETIC ANISOTROPY.
\end{abstract}

\section{Introduction}

A great deal of interest has been focused on the new magneto-optic disk materials which have a large Kerr rotation for a blue laser. Light rare earth-transition metal films such as $\mathrm{NdFeCo}$ are candidates for such materials. However, since the light rare earth-transition metal films are ferromagnetic, and their saturation magnetization (Ms) is large, the magnetization of the light rare earth-transition metal films is in-plane. To solve the problem, Gd was partly substituted for light rare earth in order to decrease the Ms. Also, the multilayer structure was introduced into the film to enhance the anisotropy[1][2]. Here, the perpendicular mag-netic anisotropy and magnetooptical properties of PrGd/FeCo multilayers (MLs) are reported. Furthermore, the origin of the enhanced anisotropy in the rare earth-transition metal MLs is discussed through the analysis of low angle $\mathrm{X}$-ray diffraction.

\section{Experimental}

$\mathrm{PrGd} / \mathrm{FeCo}$ multilayers (MLs) were prepared by the RF diode sputtering method on glass substrates like MLs in references [3][4]. The thickness ratio of rare-earth to transition metal $\left(t_{\mathrm{RE}} / t_{\mathrm{TM}}\right)$ was kept 1:1. Perpendicular magnetic anisotropy $\mathrm{Ku}$ was estimated from torque measurements and/or from the difference in magnetization curves between the easy and hard directions. Compositionally modulated structure has been confirmed by low angle X-ray diffractometry for MLs with a bilayer period thickness larger than $1 \mathrm{~nm}$. Kerr rotation and Kerr ellipticity were measured by the polarized angle modulation method from the glass side.

\section{Results and discussion}

Figure 1 shows the dependences of $\mathrm{Ku}$ and $\mathrm{Ms}^{2} / 2 \mu_{0}$ on bilayer period $\Lambda=\left(\mathrm{t}_{\mathrm{RE}}+\mathrm{t}_{\mathrm{TM}}\right)$. It is shown that $\mathrm{Ku}$ becomes the maximum at about $\Lambda=1 \mathrm{~nm}$. When the Co content was varied, the largest value of $\mathrm{Ku}$ about $30 \times 10^{4} \mathrm{~J} / \mathrm{m}^{3}$ was obtained for Co composition at around 10at.\%. From the applications point of view, namely for large $\mathrm{Ku}$ and for adequate Curie temperature of about $200^{\circ} \mathrm{C}$, the most suitable values are $1.0 \mathrm{~nm}$ for $\Lambda$ and at $10 \mathrm{at} . \%$. for Co content [4]. For these 


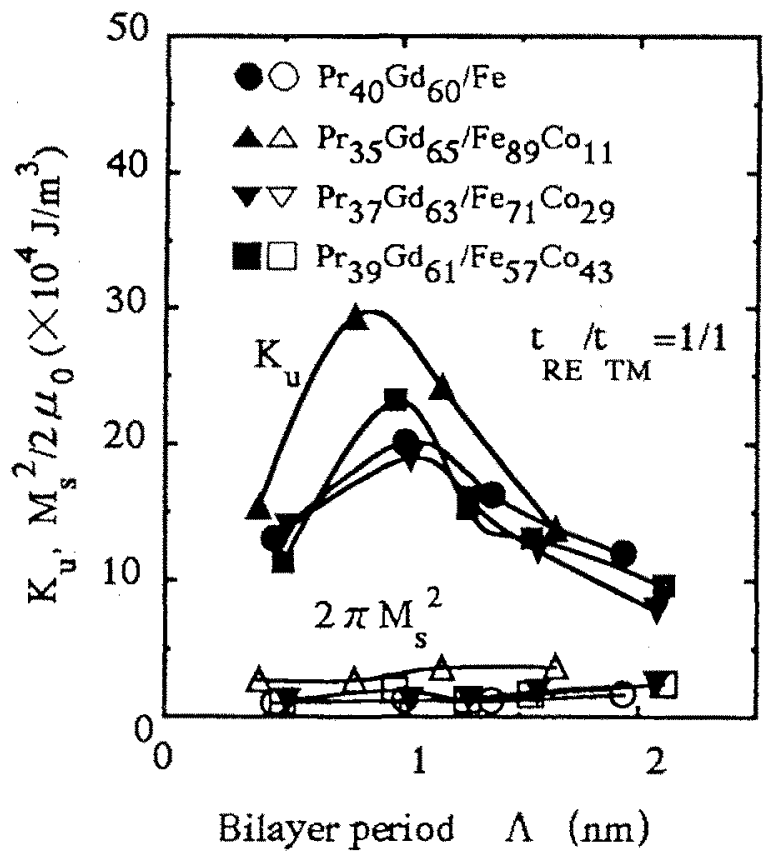

Fig. 1 Dependences of $\mathrm{K}_{\mathrm{u}}$ and $\mathrm{M}_{\mathrm{s}}^{2} / 2 \mu_{0}$ on bilayer period

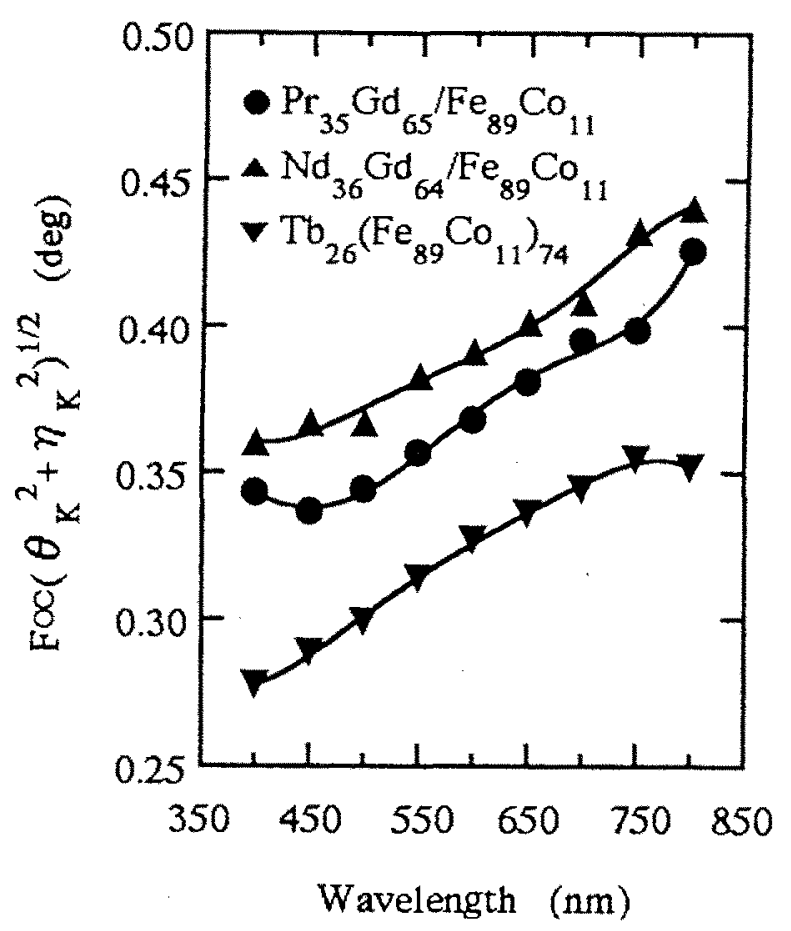

Fig.2 Spectra of figure of merit $\left(\theta_{\mathrm{K}}{ }^{2}+\eta_{\mathrm{K}}{ }^{2}\right)^{1 / 2}$ for $\mathrm{NdGd} / \mathrm{FeCo}, \mathrm{PrGd} / \mathrm{FeCo}$ and $\mathrm{Tb}(\mathrm{FeCo})$ films parameters, considerably large Kerr rotation of $0.33^{\circ}$ was also obtained at $\Lambda=1.0 \mathrm{~nm}$.

Magneto-optical figure of merits (F.M.) which is defined by $\left(\theta_{\mathbf{K}}{ }^{2}+\eta_{\mathbf{K}}{ }^{2}\right)^{1 / 2}$ are plotted in Fig.2 for $\mathrm{NdGd} / \mathrm{FeCo}$ and PrGd/FeCo MLs together with those of $\mathrm{TbFeCo}$ as a function of photon energy or wavelength. It is seen that F.M. of both MLs are 20 to $30 \%$ higher than that of conventional TbFeCo films.

To investigate the effect of the ML structure on the anisotropy, we have prepared PrGd/Fe MLs under two different sputtering conditions, keeping the composition of MLs constant. The difference is in the presence of the magnetic field above the RE target. Hereafter method 1 shows that MLs were prepared using the RE target under which Fe plate was put to bypass the magnetic flux from the magnet under the target. Method 2 shows that MLs were prepared using a RE target without the Fe plate namely the magnetron target.

Fig. 3 is shown the pattern of low angle $\mathrm{X}$-ray diffraction for the two sorts of films. The MLs shown in Figs. 3(a) and (b) were prepared by method 1 and method 2 , respectively. Comparing the two figures, we can see that the peak intensities in Fig.3 (a) are higher than those in Fig. 3 (b), especially at higher angles around 8 degree. This suggests that the interface of MLs prepared by method 1 is sharper than that of MLs by method 2 .

For the two sorts of MLs, the dependence of the anisotropy $\mathrm{Ku}$ on the bilayer period is shown in Fig.4. In both MLs, the anisotropy Ku varies in a similar way with the bilayer period, but the values of $\mathrm{Ku}$ for method 1 are considerably smaller than those by method 2 , especially at bilayer periods between 1.0 and $2.0 \mathrm{~nm}$. The demagnetization energies of both MLs are almost the 


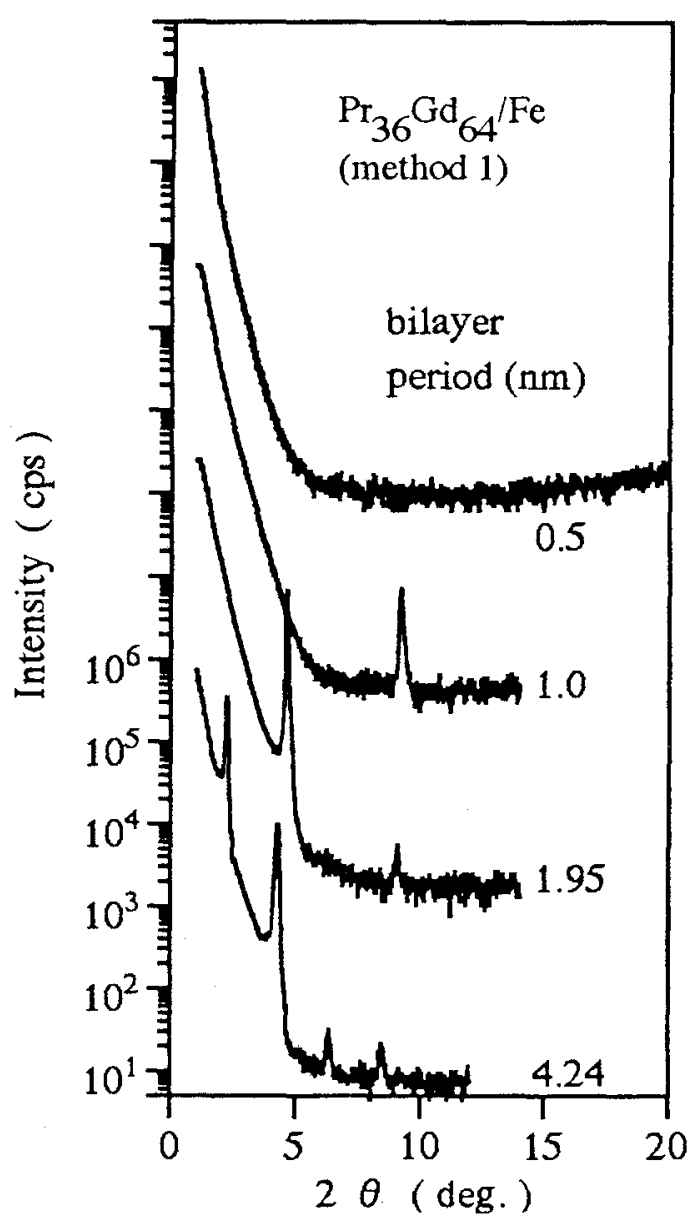

(a)

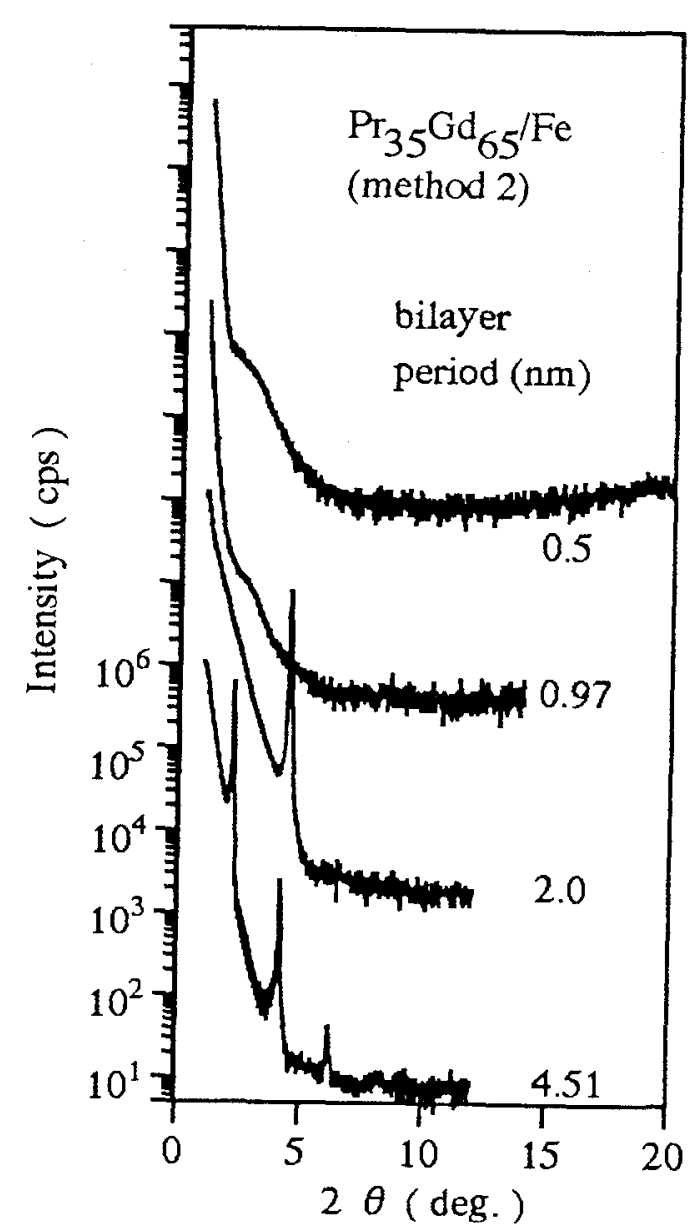

(b)

Fig.3 Pattern of X-ray diffraction of low angle for PrGd/Fe multilayer sputtered (a) by method land (b) by method 2

same for all bilayer periods examined here. We also notice, that the both sorts of MLs become in-plane magnetization for bilayer periods above $3 \mathrm{~nm}$.

From the Fig.3. and Fig.4, it can be seen that the sharper interface results in the smaller anisotropy. This fact is opposite to what we first expected. That is to say, to increase the anisotropy, we introduced the multilayer structure, and as a result, the anisotropy was enhanced by the $\mathrm{ML}$ structure. However, the anisotropy did not increase further when the interface are made sharper.

The results shown above might be explained as follows. The enhanced anisotropy in the multilayers is considered to come from the single-ion anisotropy and/or the anisotropic pseudo-dipolar-interaction, both related to non Sstate RE atoms. For the appearance of the anisotropy by such mechanisms, RE moments must be polarized through the exchange force of neighbouring $\mathrm{Fe}$ moments. Thus, to enhance the anisotropy, it is necessary that many of the nearest neighbor of $\mathrm{Fe}$ atoms exsist around RE atom, even along the direction perpendicular to the film plane. The sharp interface in multilayers may increase the uniaxial crystal field and/or the anisotropy in the atomic pair order but will decrease the number of the nearest neighbor $\mathrm{Fe}$ 


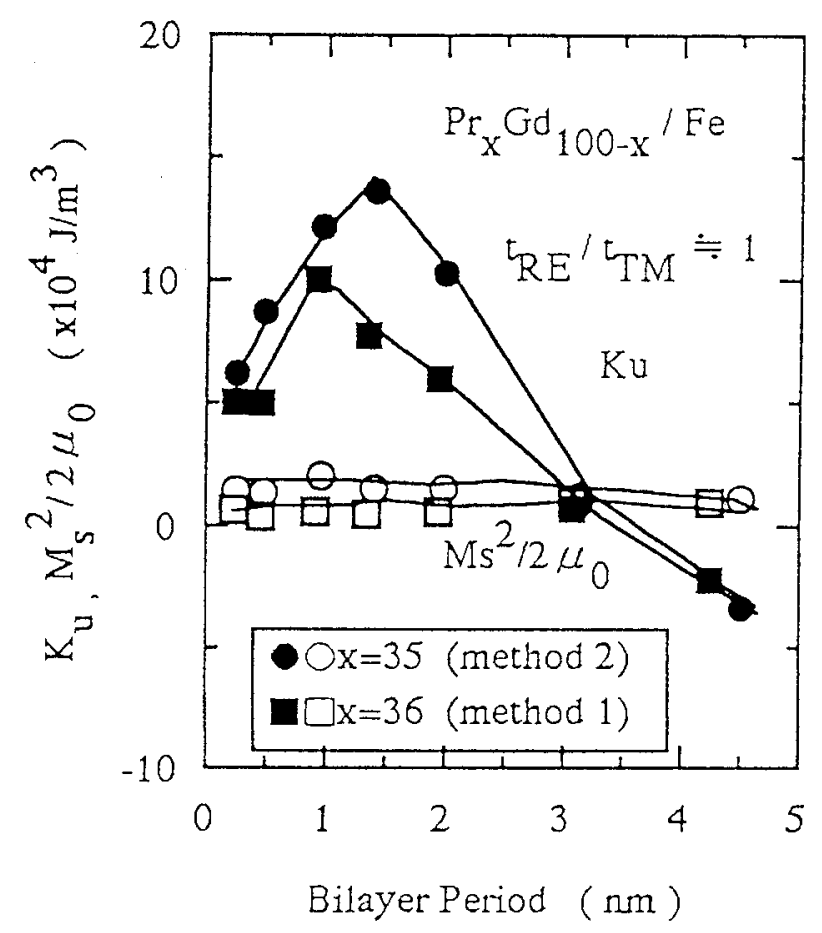

Fig.4 Dependence of $\mathrm{K}_{\mathrm{U}}$ and $\mathrm{Ms}^{2} / 2 \mu_{0}$ on bilayer period for PrGd/Fe ML prepared by two different methods (see text)

atoms when the interface is very sharp, the uniaxial anisotropy will decrease with decreasing $R E$ polarization. In this paper, we found that the maximum values of the anisotropy were obtained for the bilayer period of $1 \mathrm{~nm}$. This thickness might be determined by the competition between the increasing of anisotropic crystal field or anisotropic pair order and decreasing $R E$ polarization with decreasing $\Lambda$. For further discussion, however, we should also consider the effect of the classical dipolar energy of RE and TM moments which ordered antiferromagnetically at the interface.

\section{Conclusion}

$\mathrm{PrGd} / \mathrm{FeCo}$ multilayers exhibit perpendicular anisotropy enhanced by the ML structure. The largest value of $\mathrm{Ku}$ obtained is about $30 \times$ $10^{4} \mathrm{~J} / \mathrm{m}^{3}$ at bilayer period of $1 \mathrm{~nm}$ with Co composition around $10 \mathrm{at} . \%$. The figure merits of magneto-optic is 20 to $30 \%$ larger than conventional TbFeCo film. From analysis of low angle $X$-ray diffraction, the alloy formation at the interface seems to be closely related to the enhanced anisotropy.

\section{References}

1. N.Sato: J.Appl.Phys., 59, 2514(1986)

2.D.J.Sellmyer, Z.R.Zhao, Z.S.Shan and S.Nafils: J.Appl.Phys., 61, 4323(1987)

3. S.Tunashima, T.Ohtani, X.Y.Yu and S.Uchiyama: J. Magn. Magn. Mat. 104-107, 1021(1992)

4. X.Y.Yu, S.Tsunashima and S.Uchiyama: J. Mag. Soc. Jpn., 16, 547(1992) in Japanese 